\title{
Genomics in Hematology and Oncology Practice
}

\author{
Gordan Srkalovic \\ Herbert-Herman Cancer Center, Sparrow \\ Hospital System, Lansing, MI, USA \\ Correspondence: \\ gordan.srkalovic@sparrow.org \\ Tel.: + 15173642182 \\ Fax.: + 15173642886
}

Received: 24 April 2019

Accepted: 25 April 2019

Key Words: Genomics • Practice • Education - Future.

"What can and doesn't have to be always, at the end, surrenders to something that has to be."

Ivo Andric. Signs by the roadside (1)

Most of my colleagues when writing commentaries or overviews quote Shakespeare, and deservedly so. I have to confess, with embarrassment though, that my understanding and command of great Shakespeare's poetry is very limited. I was raised and learned to love great Russian classics and our only Nobel prize laureate in literature, Ivo Andric. Definitely, because he wrote about places and people I recognize and understand; partially because he was born and schooled in Bosnia and Herzegovina. His writing once in private conversation with my friends was described as "mighty, slow, wide river" which immediately brought in my mind Mississippi in New Orleans where my American adventure started. Power of that unstoppable force that carries everything with and in front of it always fascinated me. That is how I see progress in oncology with the introduction of genomics and prospect of precision medicine and individualized treatments. Genomics offers the potential for deeper understanding of disease pathophysiology, prognostication, identification of predictors of therapeutic responses, depth of responses, discovery of new targets for the treatment and ultimately improvement in quality of life and prospect for the cure.

In this special issue of Acta Medica Academica (AMA) we attempted to describe ways how introduction of genomics has influenced the practice of Oncology and Malignant Hematology from diagnosis to planning and coordination of care to treatment and outcomes itself. Readers will decide if we succeeded.

The main question we will have to answer in the future is: Do genomic alterations always or in the most of the cases lead to oncogenic pathway activation and how do we target multiple potential drivers and how to address development of resistance pathways in order to achieve optimal antitumor efficacy? The preliminary results from National Cancer institute Molecular Analysis for Therapeutic Choice (NCI-MATCH) trial presented at 2018 American Society of Clinical Oncology (ASCO) Annual meeting described results of 3 cohorts with no agent reaching the prespecified threshold of notable clinical activity (2). That prompted edi- 
torial in JAMA Oncology by Eckhardt and Lieu: Is precision medicine an oxymoron? (3). Authors asked very legitimate question if these results indicate that a molecularly driven agnostic approach is a failure. Their response is qualified yes and no. My reading of preliminary NCI-MATCH results is that they are exactly what they are: preliminary. These are early nascent results pointing to the fact that drugs T-DM1, AZD4547 or taselisib in patients harboring ERBB2/ HER2 amplification, FGFR alterations or PIK3CA mutations, respectively, did not provide meaningful clinical effects when addressed as isolated mutations. Nothing more or nothing less. It is true that those results also pointed to the possible shortcoming of addressing individual mutations with monotherapy. Recently published study I-PREDICT from University of San Diego presented results of targeting larger fraction of identified molecular alterations, yielding a higher "matching score" (4). This novel approach showed significantly improved disease control rates, as well as longer progression-free survival (PFS) and overall survival (OS) compared to targeting of fewer somatic alterations. Despite innovative approach and excellent results seen in this study, this is just scratching the surface. We have to keep in mind that in this study, as well as in other studies to the present, we addressed only molecular or gene alterations that have available FDA approved or experimental treatments, so called "drugable" alterations. Consequently, with increased availability of therapies targeting other possibly driving alterations, expected outcomes could, and I believe will become more and more clinically relevant.

Therefore, the articles in this issue are describing the translation of increasing knowledge and understanding of genomics to the clinical practice, having medical students, residents, fellows as well practicing hematologists and oncologists in mind. Our goal was not to give comprehensive review of present understanding of clinical genomics in Oncology and Malignant Hematology, but to provide basic premises of changes that genomics use brings to every day practice of malignant disease patient care.

We begin with an article by Trivedi et al. (5) that summarizes changing landscape of clinical practice, from empirical to evidence-based to biology-based personalized medicine. In this article authors are describing technological and intellectual advances that led to explosion of new treatments in the therapy on malignant diseases. They are describing evolution of critical thinking in oncology and development of linkage between genomic and clinical data as well as computational biology expertise required for data analysis. Ultimately, authors are painting a picture of future developments and the need for changes in education of physicians in order to fulfill promise of most appropriate care for each individualized patient.

Article by Audeh et al. (6) opens for readers almost magical world of thinking behind development of 70-gene assay MammaPrint, first FDA cleared genomic assay for breast cancer. The quality of this paper is not so much in clinical data connected with use of MammPrint, although they are very impressive. Authors in this article took "roads less travelled". They opened window into thought process and decision making behind MINDACT trial design and goals definition. Paper illustrates meticulous decision making process and weighing between what can be and what needs to be done. This paper is not relevant only for those interested in genomics in the oncological and hematological practice, but also for everybody who wants to learn about scientific process and design of research studies.

Although our understanding of molecular processes involved in the development of acute myelogenous leukemia (AML) are greatly advanced by genomic medicine, 
there are still "great unknowns". Madanat et al. (7) summarized data on commonly mutated genes and genomic pathways in AML. This data is now increasingly being used for disease classification, risk stratification, and clinical care of patients. Review highlights major updates in the World Health Organization (WHO) classification, including cytogenetic re-classification, provisional entities (AML with mutated RUNX1 and AML with BCR/ABL1) and updates to the European Leukemia Net (ELN) AML group risk stratification (RUNX1, ASXL1 and TP53). Future of treatment could be driven by complex interactions between different mutations. Assessment of minimal residual disease (MRD) also could improve risk stratification and selection of post remission therapy.

Strategic decision making required to optimize laboratory work up of lymphomas is focus of article by Shi et al. (8). Authors are discussing lure and attraction of "new shiny" tests for multiple genomic abnormalities and their significance for patient care and practical world use. Danger of over testing is real and with commercial entities pressure and financial influence we, as ultimate providers of the best care, need to have clear guidance which testing is necessary, and which will be affordable from patients' and societal view point. Authors are providing simplified algorithm for the work up of Diffuse large B-cell lymphoma (DLBCL) and High-grade B-cell lymphoma (HGBCL) which is rational and practical and could be used almost universally. As the field evolves, new tests and panels could become more affordable and clinically relevant and will become the standard of care (SOC).

Although mutational landscape of multiple myeloma (MM) seems hard to decipher due to significant heterogeneity, it is also almost perfect model case for evolution of genetic changes. MM is unique among hematological malignancies in its universal evolution from pre-malignant stages to pro- liferation of malignant cells. Due to that, significant body of literature is available examining impact of genomics on MM risk stratification and treatment. I applaud Castaneda and Baz (9) for their effort to make it easier for readers to navigate through richness of data and for summarizing them in this article. They also explained very nicely predictive value of genomic testing and possible use of this testing for evaluation of specific agents resistance development. In addition, it seems that Next generations sequencing (NGS) will become universally accepted and used tests for evaluation of Minimal Residual Disease (MRD) in MM patients. Negative MRD is now shown to correlate with better OS in MM (PRIMER study)(10).

Devitt and Dreicer (11) focus on role of genomics in Genitourinary (GU) malignancies. They described most common germ line mutations associated with prostate cancer, as well renal cell carcinoma. It is very important to emphasize that prostate cancer is one of the most heritable forms of malignancies. That is reason for recommendation for all patients with metastatic prostate cancer to be referred for genetic counselling and testing. Mutations in the genes HOXB13 and BRCA 1 or 2 have been associated with family clusters of prostate cancers. Authors also explained potential predictive role of mutation testing in the treatment of prostate cancer. Prognostic utility of mutations in PBRM1 and BAP1 in renal cell carcinoma seems to be in concordance with biological and clinical features of this disease. However, it seems that wider use of genomics in GU cancer is still in its nascent stages and more opportunities will become available in the future. That is becoming more obvious in urothelial cancers, as molecular subtyping using gene expression profiling has emerged as a prognostic and predictive tool.

Use of genomic testing completely changed landscape of lung cancer treatments. Body of literature dealing with this 
topic is growing exponentially and Ankur Parikh (12) summarized present state of knowledge and open questions waiting for answers. Presently, defining treatment for lung cancer is almost impossible without analysis of targetable genes altered in nonsmall cell cancer (NSCLC). Treating patients without knowledge of EGFR, ALK, ROS1, BRAF, MET, HER2, RET and NTRK1 mutations is now considered far outside of SOC. Big data analysis and long term results from ongoing genomics based clinical trials will open other, at the present, uncovered avenues for more effective treatments of this deadly disease.

SOC treatments are also rapidly evolving in the arena of Gynecologic (GYN) malignancies. Maurie Markman (13) reviewed present state of art approaches to treatment of ovarian cancer and changing landscape based on BRCA mutations. In addition multiple other potentially "driver" mutations (PIK3CA, ADAMTS, DICER1, BRAF, KRAS, ARIDA1A and others), although presently still not targetable or "drugable" could become soon very effective targets with potentially less toxicity and better clinical outcomes. Use of check point inhibitors is becoming more and more standard in the treatment of GU malignancies, and genomic analysis could potentially define patients who are good candidates for these treatments.

Saadeh et al. (14) took task to review very important topic of application of precision medicine in oncology pharmacy practice. This topic is not frequently covered in oncology literature and, in my opinion, this is very important contribution to education of practicing oncologists. Explosion of new malignancies targeting medications is creating new challenges and new opportunities for clinical pharmacists, particularly those specializing in Pharmacogenomics (PGx). This component of precision medicine is based on polymorphisms and strongly impacts drugs selection and dosing. Although new medications are very effective, they bring potentially new and different toxicities that could be reduced and minimized by using pharmacogenomics-based dosing recommendations. It is very important to emphasize critical role of clinical pharmacists in supporting multidisciplinary approach to the care of patients with cancers.

Very interesting case report describing crucial role of comprehensive genomic profiling in the treatment of patient with high stage uterine mesenchymal tumor is presented by Lee et al. (15). Testing showed to be essential for establishing correct diagnosis as well as uncovering until then unrecognized ALK mutation used subsequently as target for effective treatment. This shows how effective analysis can change life of oncology patients, one patient at time.

Last, but not least is genomics practice article by Trivedi et al. (16). Oncology patient whose tissue is analyzed by next generation sequencing (NGS) can show targetable mutations or be candidates for genomics based clinical trial. However, significant number of patients will not fit into either of these groups and additional options need to be investigated. That is where Molecular Tumor Board (MTB), multidisciplinary panel discussion comes into play opening other possible avenues based on in depth analysis of growth pathways and role of mutated genes in signal transduction. In this article, results of MTB in mid-size cancer center are described. It illustrates the advantages provided to patients by finding treatments when no other options are available, as well as missed opportunities based on physicians' and patients' preferences or biases. It also emphasizes the need for education of patients, physicians and general public about incredible advantages and possible shortcomings of genomics testing and concepts of precision medicine. I honestly hope this issue of AMA could help making that educational task easier. 
Acknowledgment: Author would like thank Miss. Kathryn Edwards and Dr. Harsha Trivedi for assistance with manuscript preparation.

Conflict of Interest: The author declares that he has no conflict of interest.

\section{References}

1. Andric I. Signs by the roadside. Translated by Stanislava Lazarević. 1st ed. Belgrade: Sezam book; 2015.

2. Krop IE, Jegede O, Grilley-Olson JE, Lauring JD, Hamilton SR, Zwieber JA. Results from Molecular Analysis for Therapy Choice (MATCH) arm I: taselisib for PIK3CA-mutated tumors. J Clin Oncol. 2018:36(Suppl 15):abstract 101.

3. Eckhardt SG, Lieu C. Is precision medicine an oxymorone? JAMA Oncol. 2018 Nov 8. [Epub ahead of print].

4. Sicklick JK, Kato S, Okamura R, Schwaederle M, Hahn ME, Williams CB, et al. Molecular profiling of cancer patients enables personalized combination therapy: the I-PREDICT study. Nat Med. 2019;25(5):744-50. Epub 2019 Apr 22.

5. Trivedi H, Kling HM, Treece T, Audeh W, Srkalovic G. Changing Landscape of Clinical-Genomic Oncology Practice. Acta Med Acad. 2019;48(1):6-17.

6. Audeh W, Blumencranz L, Kling HM, Trivedi H, Srkalovic G. Prospective Validation of a Genomic Assay in Breast Cancer: The 70-gene MammaPrint Assay and the MINDACT Trial. Acta Med Acad. 2019;48(1):18-34.

7. Madanat YF, Kalaycio, ME, Nazha A. Advances in Acute Myeloid Leukemia Genomics, Where Do We Stand in 2018? Acta Med Acad. 2019;48(1):3544.
8. Shi M, Dao L, Jevremovic D. Laboratory WorkUp of Chronic B-Cell Lymphoid Malignancies - A Value-Based Approach. Acta Med Acad. 2019;48(1):45-56.

9. Castaneda O, Baz R. Multiple Myeloma Genomics - A Concise Review. Acta Med Acad. 2019;48(1):57-67.

10. Hahn TE, Wallace PK, Fraser R, Fei M, Tario JD, Howard A, et al. Minimal Residual Disease (MRD) Assessment before and after Autologous Hematopoietic Cell Transplantation (AutoHCT) and Maintenance for Multiple Myeloma (MM): Results of the Prognostic Immunophenotyping for Myeloma Response (PRIMeR) Study. Biology of Blood and Marrow Transplantation. 2019;25(3 Suppl):S4-6.

11. Devitt ME, Dreicer R. Evolving Role of Genomics in Genitourinary Neoplasms. Acta Med Acad. 2019;48(1):68-77.

12. Parikh AR. Lung Cancer Genomics. Acta Med Acad. 2019;48(1):78-83.

13. Markman M. Genomic-Based Therapy of Gynecologic Malignancies. Acta Med Acad. 2019;48(1): 84-9.

14. Saadeh C, Bright D, Rustem D. Precision Medicine in Oncology Pharmacy Practice. Acta Med Acad. 2019;48(1):90-104.

15. Lee J, Singh A, Ali SM, Lin DI, Klempner SJ. TNS1-ALK Fusion in a Recurrent, Metastatic Uterine Mesenchymal Tumor Originally Diagnosed as Leiomyosarcoma. Acta Med Acad. 2019;48(1):115-20.

16. Trivedi H, Acharya D, Chamarthy U, Hamdan M, Herman J, Srkalovic G, et al. Implementation and Outcomes of a Molecular Tumor Board at Herbert-Herman Cancer Center, Sparrow Hospital. Acta Med Acad. 2019;48(1):105-15. 